\title{
SFTA3, a novel protein of the lung: three- dimensional structure, characterisation and immune activation
}

\author{
Martin Schicht ${ }^{1,7}$, Felix Rausch ${ }^{1,7}$, Susetta Finotto², Martina Mathews ${ }^{2}$, \\ Anja Mattil2, Melanie Schubert ${ }^{2}$, Beate Koch², Maximilian Traxdorf ${ }^{3}$, \\ Christopher Bohr ${ }^{3}$, Dieter Worlitzsch ${ }^{4}$, Wolfgang Brandt ${ }^{5}$, Fabian Garreis ${ }^{1}$, \\ Saadettin Sel ${ }^{6}$, Friedrich Paulsen ${ }^{1,8}$ and Lars Bräuer ${ }^{1,8}$
}

\begin{abstract}
Affiliations: 'Institute of Anatomy, Dept II, Friedrich Alexander University Erlangen-Nuremberg, Erlangen, Germany. ${ }^{2}$ Dept of Molecular Pneumology, Friedrich Alexander University Erlangen-Nuremberg, Erlangen, Germany. ${ }^{3}$ Dept of Otorhinolaryngology, Head and Neck Surgery, Friedrich Alexander University ErlangenNuremberg, Erlangen, Germany. ${ }^{4}$ Dept of Hygiene of the Medical Faculty, Martin Luther University of HalleWittenberg, Halle (Saale), Germany. ${ }^{5}$ Dept of Bioorganic Chemistry, Leibniz Institute of Plant Biochemistry, Halle, Germany. ${ }^{6}$ Dept of Ophthalmology, Ruprecht-Karls University Heidelberg, Heidelberg, Germany. ${ }^{7}$ These two authors contributed equally to this work. ${ }^{8}$ These two authors contributed equally to this work.
\end{abstract}

Correspondence: Martin Schicht, Dept of Anatomy II, University of Erlangen-Nuremberg, Universitätsstraße 19, 91054 Erlangen, Germany. E-mail: martin.schichtdanatomie2.med.uni-erlangen.de

ABSTRACT The lung constantly interacts with numerous pathogens. Thus, complex local immune defence mechanisms are essential to recognise and dispose of these intruders. This work describes the detection, characterisation and three-dimensional structure of a novel protein of the lung (surfactantassociated protein 3 (SFTA3/SP-H)) with putative immunological features.

Bioinformatics, biochemical and immunological methods were combined to elucidate the structure and function of SFTA3. The tissue-specific detection and characterisation was performed by using electron microscopy as well as fluorescence imaging.

Three-dimensional structure generation and analysis led to the development of specific antibodies and, as a consequence, to the localisation of a novel protein in human lung under consideration of cystic fibrosis, asthma and sepsis. In vitro experiments revealed that lipopolysaccharide induces expression of SFTA3 in the human lung alveolar type II cell line A549. By contrast, the inflammatory cytokines interleukin (IL)-1 $\beta$ and IL-23 inhibit expression of SFTA3 in A549. Sequence- and structure-based prediction analysis indicated that the novel protein is likely to belong to the family of lung surfactant proteins.

The results suggest that SFTA3 is an immunoregulatory protein of the lung with relevant protective functions during inflammation at the mucosal sites.

@ERSpublications

SFTA3: a novel lung protein with putative protective and immunological functions during inflammation in lung diseases http://ow.ly/tsnne

This article has supplementary material available from erj.ersjournals.com

Received: Oct 152013 | Accepted after revision: Jan 252014 | First published online: April 172014

Support statement: This study was supported by the German Research Foundation (DFG, Programme Grants 1329/12-1 BR and BR 3681/2-1), European Grant PreDicta, "Post-infectious immune reprogramming and its association with persistence and chronicity of respiratory allergic diseases" at the UK-ER, Erlangen and the Wilhelm Roux Programme, Halle, Germany (Programme Grants FKZ 17/20).

Conflict of interest: None declared.

Copyright @ERS 2014 


\section{Introduction}

The lung surface is lined with a complex mixture of phospholipids and proteins known as pulmonary surfactant. It is essential for normal respiratory mechanics and reduces the surface tension of the air-liquid interface and, as a consequence, prevents collapse of the alveoli. A lack of surfactant leads to respiratory failure [1]. Beyond this function, the proteins of the pulmonary surfactant, surfactant proteins (SP), contribute to the innate immune defence against inhaled pathogens and moreover act as anti-inflammatory substances [2]. Lung SPs are mainly secreted by alveolar type II cells.

Four different lung SPs have been described to date: two hydrophobic proteins, SP-B and SP-C, and two collectin-like proteins, SP-A and SP-D. These proteins have been described in detail in relation to their lung surface activity and immunological defence function [2].

SP-A and SP-D are representatives of the C-type lectin family, which according to current understanding are able to bind to specific carbohydrates of bacteria, protozoans, fungi and viruses [3]. This function facilitates opsonisation of and accelerates immune defence reactions towards these microorganisms [4].

In contrast to SP-A and SP-D, the small and extremely hydrophobic proteins SP-B and SP-C are essential components during formation of surfactant monolayers and stabilisation of air-fluid interfaces $[5,6]$. Similar to SP-A and SP-D, the presence of SP-B and SP-C has already been demonstrated in a variety of tissues and humours, including tissues of the nasolacrimal apparatus and ocular surface, in tear fluid, in salivary glands, in the gingiva and nasal mucosa [7-10]. Due to the homology to amoebapores, an immunological function of SP-B has been also been discussed [11]. Recent publications demonstrate the role of SP-B in the activation of alveolar macrophages in the innate immune response in the lung [11]. Recently, another new surfactant protein SP-G has been identified and described [12]. SP-G has physicochemical properties similar to SP-B and SP-C. The possibility of interactions with lipid systems and a potential surface-regulatory function of SP-G have been discussed. Alveolar macrophages constitute an important defence against infection because they clear pathogens. Maintaining normal and consistent amounts of alveolar surfactant is in part dependent on clearance of surfactant by alveolar macrophages [13]. While working with the four known SPs, our attention was attracted to another putative surfactant protein. It was theoretically identified by bioinformatics and named surfactant protein $\mathrm{H}$ (SP-H) or surfactantassociated protein 3 (SFTA3) [14]. The official gene symbol is SFTA3 ("Surfactant associated 3"; HUGO Nomenclature Committee). This putative SP shows no significant sequential or structural similarities to other SPs or other known proteins in general. The protein (SFTA3 or SP-H) is encoded on the human chromosome 14, its primary translation product consists of 94 amino acid residues with a molecular weight of $\sim 10 \mathrm{kDa}$. The aim of the study was the first detection and characterisation of this novel protein of the lung.

\section{Methods summary}

All tissue samples were obtained from cadavers donated to the Dept of Anatomy and Cell Biology, Martin Luther University of Halle-Wittenberg, Halle, Germany. Bronchoalveolar lavage (BAL) and sputum samples were obtained from patients of the Dept of Otorhinolaryngology-Head and Neck Surgery, Friedrich Alexander University Erlangen-Nuremberg, Erlangen, Germany and of the Dept of Hygiene, Martin Luther University of Halle-Wittenberg, in accordance with the Declaration of Helsinki, the study was approved by the Institutional Review Board (Martin Luther University Halle-Wittenberg) as well as the responsible ethics committee. The acquisition of BAL and cystic fibrosis (CF)-sputum is further described in the online supplementary material.

RNA preparation, cDNA synthesis and PCR analysis was performed using the primers (see the online supplementary material) previously described by SCHICHT et al. [10]. Quantitative real-time RT-PCR using cultivated as well as stimulated A549 cells was performed as previously described by REPPERT et al. [15]. Western blot, ELISA (USCN Life Science, Wuhan, PRC) and immunohistochemical detection of the protein was performed as described by SCHICHT et al. 2013 [10] (see the online supplementary material). The protocol for immunoelectron transmission electron microscopy, as well as for cutting $50 \mathrm{~nm}$ ultrathin sections using a transmission electron microscope (Zeiss 900; Carl Zeiss Meditec AG, Jena, Germany), is described in the online supplementary material.

The three-dimensional structural model for SP-H was obtained from the Robetta online server (http:// robetta.bakerlab.org). The prediction of post-translational modifications was carried out using various online tools (online supplementary material) and the model stability was assessed by YASARA simulations (www.yasara.org/). The molecular dynamics (MD) simulations in the lipid environment were performed with the GROMACS program package (www.gromacs.org/). These modelling steps were performed as described in detail by RAUSCH et al. [12]. 


\section{Results and discussion}

Characteristics of the human SFTA3 gene and expression of specific RNA amplification products SFTA3 is a single copy gene on chromosome $14 \mathrm{q} 13.3$. The transcript comprises $1500 \mathrm{bp}$ with 5 exons in the total genomic region (fig. 1a). SFTA3 is positioned close to the NKX2 genes. Different tissues were analysed by PCR and SP-H mRNA was shown to be expressed especially at the mucosal sites. The human lung alveolar type II cell line A549 also expressed SP-H mRNA (fig. 1b and c). The detected PCR bands are in accordance with the expected sequences for SFTA3 in gene bank data.

\section{SP-H protein structure model and post-translational modifications}

A structural model for SP-H was created by the ab initio structure prediction server Robetta. After minor refinements, the model showed a native-like fold and very good stereochemical quality according to the quality assessment tools PROSA II (https://prosa.services.came.sbg.ac.at/prosa.php) and PROCHECK (www.ebi.ac.uk/thornton-srv/software/PROCHECK/). A $20 \mathrm{~ns}$ MD simulation in a water box showed the stability of the final model. Scanning the SP-H sequence with sequence-based prediction tools for posttranslational modifications (PTMs) gave the results summarised in table 1. All the predicted PTMs were manually added to the SP-H model and again a $20 \mathrm{~ns}$ MD simulation showed the stability of the modified model. While the PTMs had nearly no effect on the model stability, they influenced protein surface properties. They could form polar or hydrophobic areas (depending on the PTM type) on the protein surface with a significant impact on water solubility or the protein-lipid interaction potential of SP-H. Two stable SP-H

a)
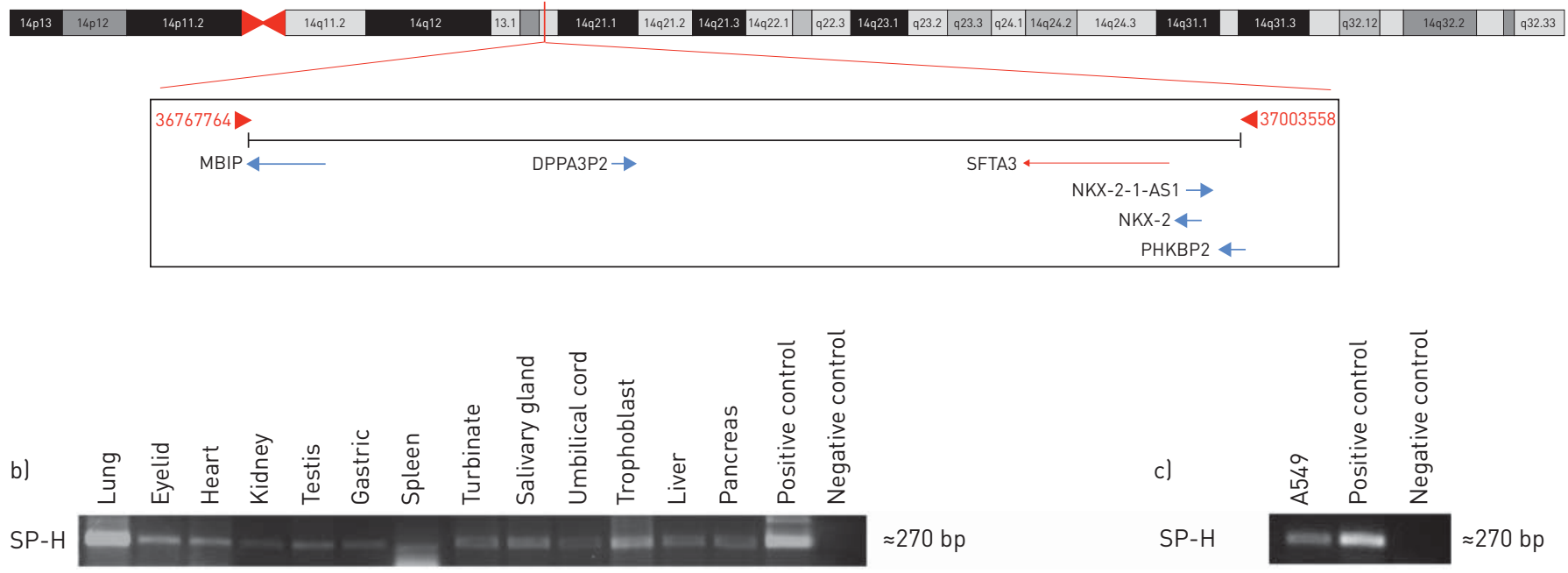

d)

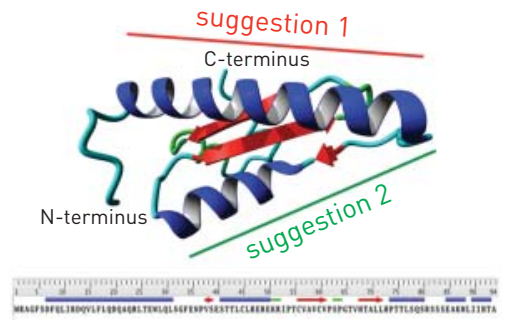

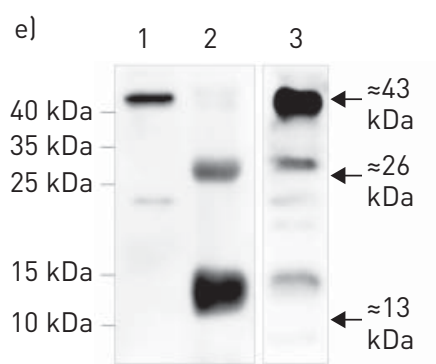

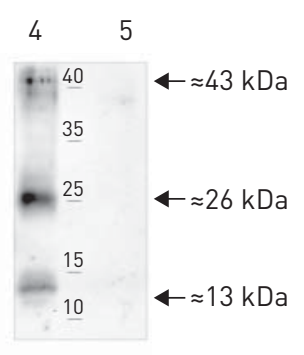

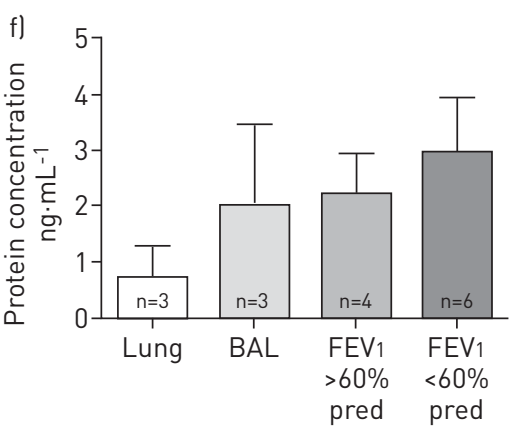

FIGURE 1 Detection and characterisation of SFTA3 and the expressed protein SP-H (surfactant protein H). a) Gene map showing the position of SFTA3 on chromosome 14 q13.3. Expression of specific SP-H mRNA amplification products in b) tissues and c) the human lung alveolar type II cell line A549. Negative controls were carried out without template cDNA and positive controls used plasmid DNA carrying the open reading frame for the respective SP-H. d) Threedimensional structure model of SP-H obtained by the ab initio modelling server Robetta (http://robetta.bakerlab.org). Only the protein backbone is shown. Blue: $\alpha$-helices; red: $\beta$-sheets; cyan/green: turns and random elements. The labels indicate suggestion 1 (red) and suggestion 2 (green) for antibody production. e) Western blot analysis with an anti-SP-H antibody using A549 cells (lane 1), lung tissue (lane 2), bronchoalveolar lavage (BAL) (lane 3), lung tissue without preincubation (lane 4) and lung tissue after pre-incubation with antigen-peptide (lane 5). $\mathrm{f}$ ) ELISA quantification of SP-H in lung tissue ( $\mathrm{n}=3$ ), BAL ( $\mathrm{n}=3$ ) and in cystic fibrosis sputum $(\mathrm{n}=10)$ with forced expiratory volume in $1 \mathrm{~s}(\mathrm{FEV} 1)>60 \%$ predicted or FEV1 $<60 \%$ pred. Mean values of SP-H for lung tissue $0.7 \mathrm{ng} \cdot \mathrm{mg}^{-1}$, BAL $2.0 \mathrm{ng} \cdot \mathrm{mg}^{-1}$, cystic fibrosis sputum with FEV1 $>60 \%$ pred $2.2 \mathrm{ng} \cdot \mathrm{mg}^{-1}$ and with FEV $1<60 \% \mathrm{pred} 3.1 \mathrm{ng} \cdot \mathrm{mg}^{-1}$. 


\begin{tabular}{|c|c|c|c|c|c|c|c|c|c|c|c|c|c|c|}
\hline $\begin{array}{l}\text { SP-H sequence } \\
\text { position }\end{array}$ & Ser32 & Ser39 & Cys45 & Thr55 & Cys56 & Thr66 & Thr69 & Thr75 & Ser78 & Ser80 & Ser82 & Ser83 & Ser84 & Thr93 \\
\hline Modification & PHOS & O-GLC & PALM & PHOS & PALM & $0-G A L$ & $0-G A L$ & $0-G A L$ & $0-G L C$ & PHOS & PHOS & PHOS & PHOS & O-GAL \\
\hline
\end{tabular}

PHOS: phosphorylation; O-GLC: O-linked glycosylation with GlcNAc (N-acetylglucosamine); PALM: palmitoylation; 0-GAL: 0-linked glycosylation with GalNAc ( $N$-acetylgalactosaminel.

models were obtained which could be used for more sophisticated simulation studies. For more information about the modelling process, the protein model and PTMs, please see the online supplementary material.

\section{Generation of a specific SP-H antibody and SP-H localisation in lung tissue}

With the help of the protein structure model obtained for SP-H, two potential antigen sequences could be identified (fig. 1d). The first suggestion covers the very stable $\mathrm{N}$-terminal $\boldsymbol{\alpha}$-helix from position 7 to 31 (DFQLIRDQVLFLQDQAQRLTEWLQL) and the second suggestion comprises the amino acid positions 35 to 51 (ENPVSESTTLCLREREK). Both regions contain various amino acids with functional groups, which would allow specific binding of an antibody. But whereas there are no predicted PTMs within the sequence of the first suggestion, the predicted $O$-glycosylation at position 39 and palmitoylation at position 45 could interfere with a proper binding of the antibody to the second suggested area. Furthermore, the spatial proximity of the palmitoylation on position 56 could cause steric hindrance. Therefore, the antibodies for SP-H were produced solely based on a shorter version of the first suggestion (DFQLIRDQVLFLQDQAQ). A BLAST-search (http://blast.ncbi.nlm.nih.gov/Blast.cgi) with this peptide sequence showed no hits for another human protein sequence. Therefore, it was considered as unique in the human proteome.

Anti-peptide antibodies were generated against a specific region of the human SP-H sequence (CDFQLIRDQVLFLQDQAQE). The peptide was synthesised by SeqLab (Göttingen, Germany). The specificity of the antibody was verified by Western blot analysis. The specificity of the resulting antibody was tested with protein from lung tissue and BAL $(30 \mu \mathrm{g})$ (fig. 1e). The purified antibody shows distinct protein bands in lung for SP-H at $13 \mathrm{kDa}, 26 \mathrm{kDa}$ and $43 \mathrm{kDa}$. Lung tissue was used as a specific positive control for SPs. The analysis of BAL showed distinct bands at $13 \mathrm{kDa}, 26 \mathrm{kDa}$ and $43 \mathrm{kDa}$, no distinct bands above $43 \mathrm{kDa}$ could be observed.

The quantification of SP-H in lung and BAL show a concentration between $0.06-1.83 \mathrm{ng} \cdot \mathrm{mg}^{-1}$ in lung $(\mathrm{n}=3)$ and between $0.15-4.87 \mathrm{ng} \cdot \mathrm{mg}^{-1}$ in BAL $(\mathrm{n}=3)$ (fig. 1f). The analysis of sputum from CF patients showed a small, nonsignificant increase in SP-H with severity of CF (stratified by forced expiratory volume in $1 \mathrm{~s}(\mathrm{FEV} 1)$ ) (fig. 1f). The concentration of SP-H varied between $0.25-3.47 \mathrm{ng} \cdot \mathrm{mg}^{-1}$ for $\mathrm{FEV} 1>60 \%$ predicted CF sputum and $0.78-6.51 \mathrm{ng} \cdot \mathrm{mg}^{-1} \mathrm{FEV} 1<60 \%$ pred CF sputum.

The quantification of SP-H in lung tissue and BAL shows different protein concentrations, but they are similar to the protein concentrations observed for the known SPs. It is known that the protein concentration is very variable and influenced by infection, smoking and by respiratory effects. The detection of SP-H with in BAL indicates that the cells of the lung secrete the protein. The ELISA analysis with samples from CF patients shows a no regulation effect of SP-H in severe CF. The result is in accord with microarray date and a big variance between the SP-H mRNA concentrations in dependence of CF [16]. It seems that the different results from distinct time points of infection, drug treatment and patient age, Similar effects are known from CF where the protein concentration of SP is increased on the one hand or down regulated on the other hand [17].

Interestingly, SFTA3 seems to be involved in the neonatal respiratory distress syndrome with intact NKX2-1 gene [18].

\section{Recombinant expression of SFTA3 protein and validation of the antibody}

The specificity of the antibody used was validated by performing a pre-incubation with the antigen-peptide and by using recombinant expression of SFTA3 protein. For pre-incubation, the antigen-peptide was incubated for $1 \mathrm{~h}$ at room temperature with the anti-SP-H antibody to block the antigen-binding-site. The blocked antibody was subsequently utilised for Western blots as well as immunohistochemistry to prove the specificity. No antibody reactivity against SFTA3 protein within lung tissues could be observed (fig. 1e). Furthermore, SP-H was overexpressed using Escherichia coli and a pBad TOPO TA high copy number plasmid (online supplementary material). The expressed protein carries an N-terminal V5-epitope and a polyhistidine-tag at the C-terminus. The Western blot (online supplementary fig. S1) using the SP-H 
antibody displays distinct bands at $\sim 18 \mathrm{kDa}$ and $\sim 43 \mathrm{kDa}$. After subtracting the molecular weight of the V5-epitope and the polyhistidine-tag the bands of the recombinant protein are in accordance to the molecular weight of the SFTA3 detected in tissues and BAL. Performing Western blot analysis using an antiV5 antibody the same distinct protein bands were detected, indicating a specific reaction of the anti-SP-H antibody on the one hand and proper recombinant expression on the other hand.

\section{Simulation of the protein models in a lipid environment}

The SP-H model without PTMs was calculated for $50 \mathrm{~ns}$ in four independent MD simulations and showed a stable root mean square deviation (RMSD) after approximately 10 ns. To produce different protein-lipid interaction scenarios, the orientation of the protein with respect to the lipid surface was manually altered for each simulation start. In all cases, the model started to interact with the dipalmitoylphosphatidylcholine (DPPC) monolayer after 2-10 ns. In the simulation with the highest interaction potential, the protein was fixed on the lipid surface mostly by interactions of the polar residues of $\alpha$-helix spanning residues $7-31$ (fig. 2b). The progression of the RMSD (fig. 2a, black plot) underlines the stability of this complex. The hydrophobic amino acids of the helix caused a hollow on the monolayer surface, which enabled immersion of the C-terminal parts of the protein below the head group region. This reduced the lipid ordering and layer stability, as indicated by the increased fluctuations in the area per lipid plot (fig. 2c, black plot). For the SP-H model with attached PTMs, MD simulations with the same setup were performed, and the results showed different interaction sites. In the most stable simulation, the $\alpha$-helix spanning residues $7-31$ was positioned in a nearly perpendicular orientation (fig. 2d). Only the N-terminus (residues 1-6) and major parts of the C-terminus (residues 75-94) were interacting with the lipids. Whereas the unmodified model showed an immersion into the lipid layer involving mainly hydrophobic interactions of the C-terminus, the interactions of the modified model were mostly driven by hydrogen bonds. The palmitoylations were also in close proximity to the monolayer surface, but were not interacting with lipids. They were flexibly attached to the protein, forming hydrophobic spots on the surface, which were shielded from the surrounding water during the MD by hydrophilic modifications of the flexible loop spanning residues 75-84. The whole protein structure was stable during the contact formation between protein and monolayer (fig. 2a, red RMSD plot). However, the interaction may not have reached its final state at the end of the simulation,
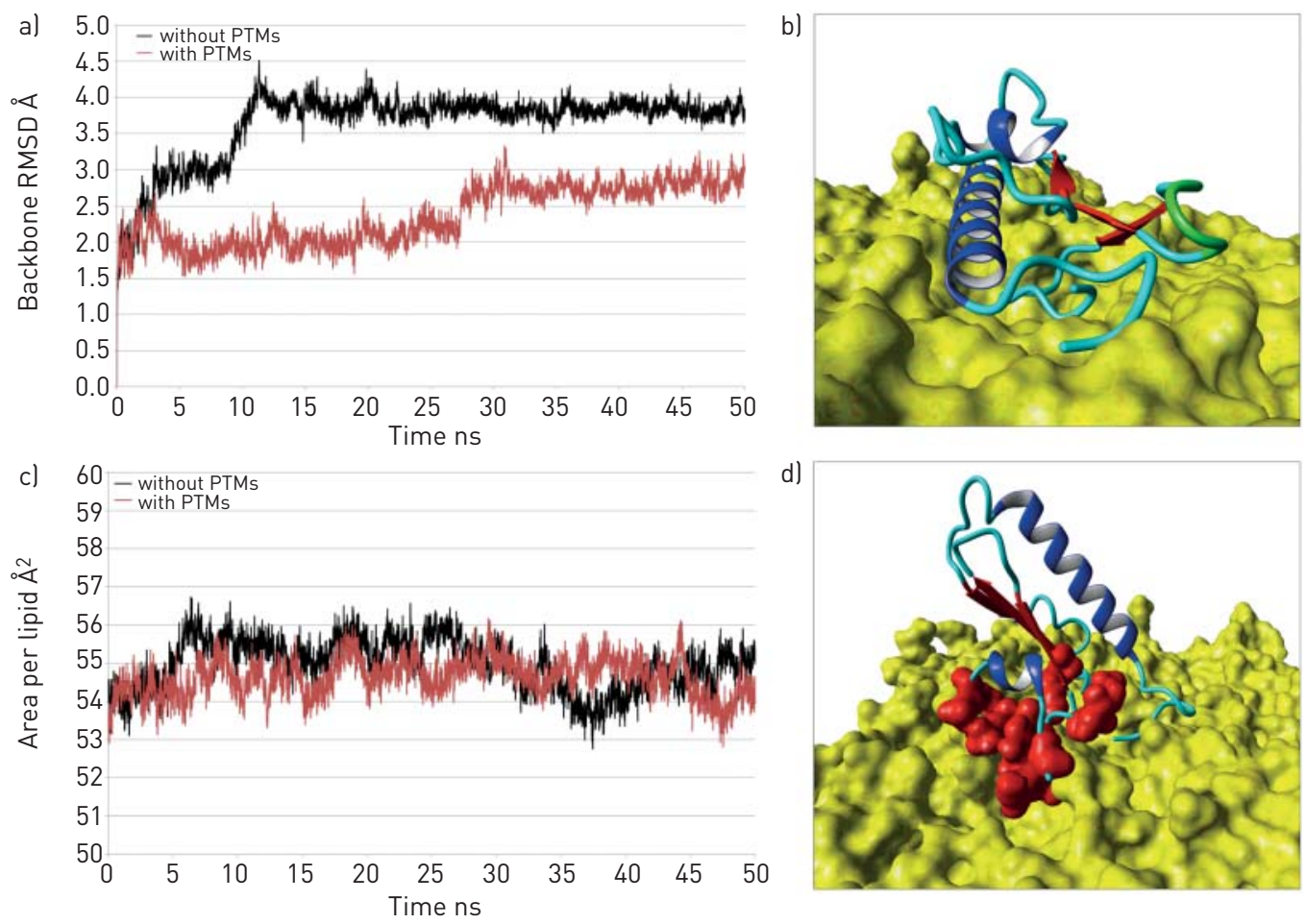

FIGURE 2 a) Root-mean-square deviation (RMSD) plot, c) area per lipid plot and structures after molecular dynamics simulations $(50 \mathrm{~ns})$ of the SP-H (surfactant protein $\mathrm{H}$ ) protein model b) without and d) with post-translational modifications (PTMs) in the presence of a DPPC monolayer. In both the SP-H protein model with and without PTMs a stable protein structure and lipid surface is indicated. The protein in b) and d) is shown in ribbon presentation $(\alpha$-helices: blue; $\beta$-sheets: red; turns: green; coil: cyan) and the lipids with a yellow van der Waals surface. PTMs with tight interactions to lipids are shown as a red van der Waals surface in (d). 
because an influence on monolayer characteristics or stability was not evident from the very stable area per lipid plot (fig. 2c, red plot). With a longer MD simulation, a deeper immersion and interactions, especially involving the attached palmitoylations, with significant effect on the lipid system could be observed. Nevertheless, the simulations showed that SP-H, without and with PTMs, has the potential to interact with lipid systems (fig. 2b-d).

\section{Detection and distribution of SP-H in lung and in A549 cells by means of immunohistochemistry and} immunogold electron microscopy

All investigated lung tissue samples showed antibody reactivity against SP-H (fig. 3a). Within lung tissue, it could be demonstrated that SP-H is distributed especially in the alveolar cells (I and II), in alveolar macrophages and is cytoplasmic in the epithelium.

The cellular distribution is similar to the known SPs; it is known that SPs are localised in the cytosol of epithelial cells as granular structures $[9,19]$. SP-H is secreted as a layer in the bronchioles (fig. 3a) and also showed reactivity in Western blot experiments on BAL samples (fig. 1e and f). Immunocytochemical investigations on A549 cells showed that SP-H is localised on the outer cell membrane (fig. 3b). The results obtained from electron microscopy indicate that the protein is also secreted and presented on the membrane of the ciliated cells, as demonstrated by positive immunoreactivity of the gold-labelled

a)
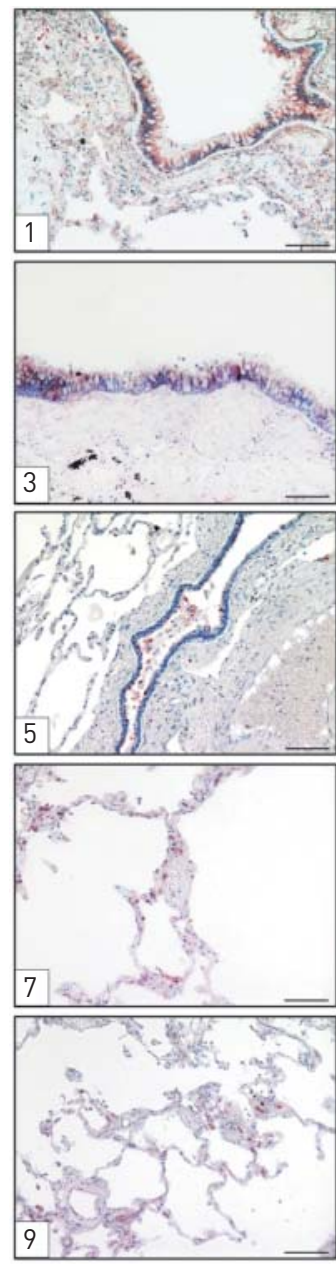
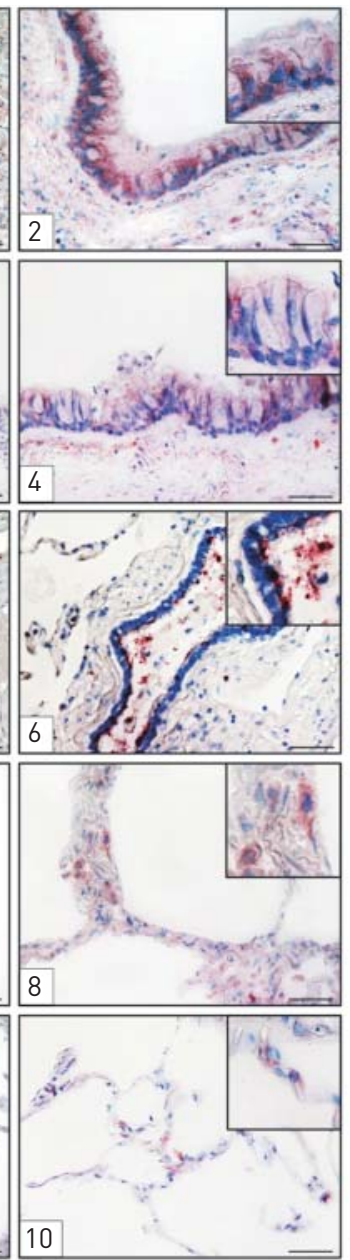

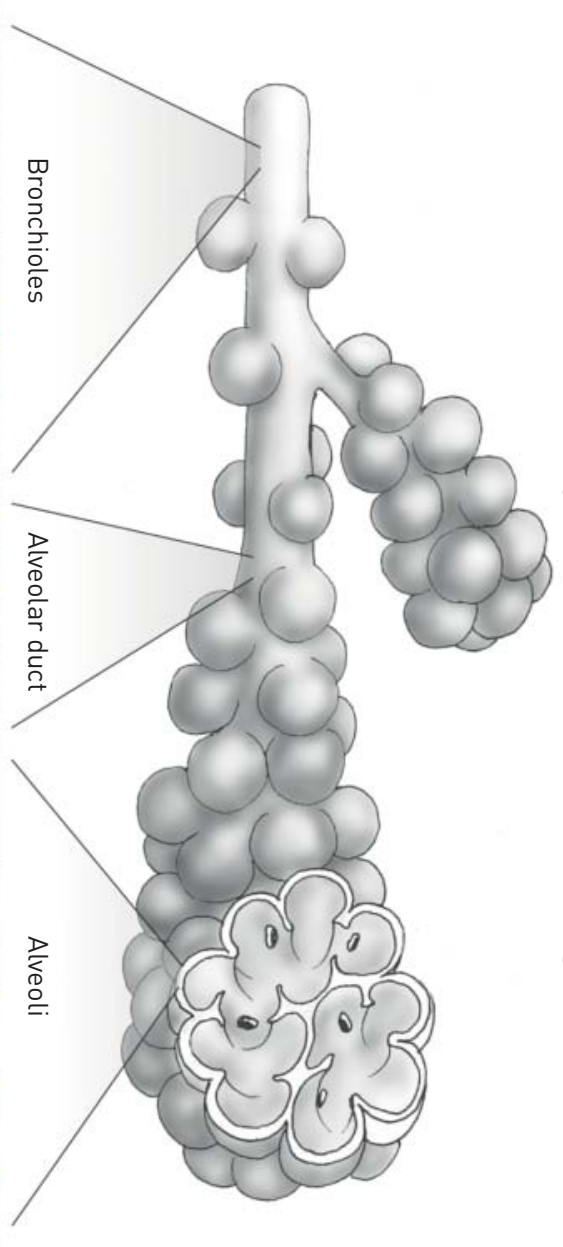

b)

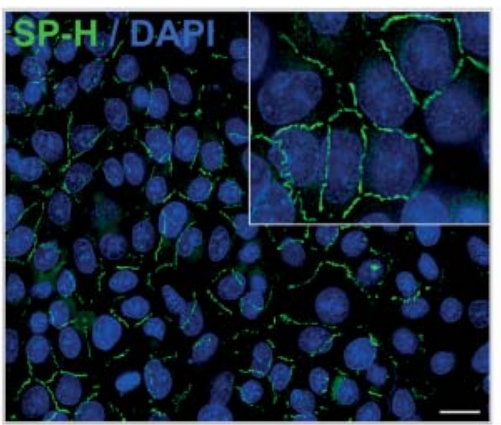

c)

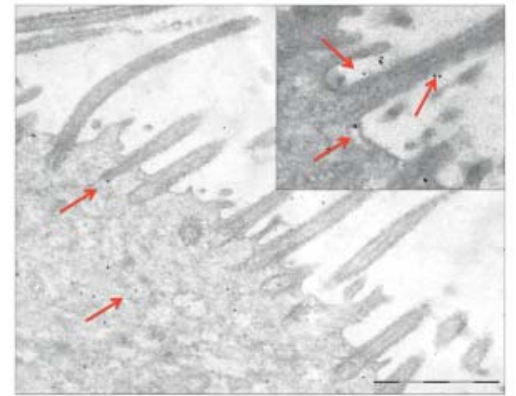

d)

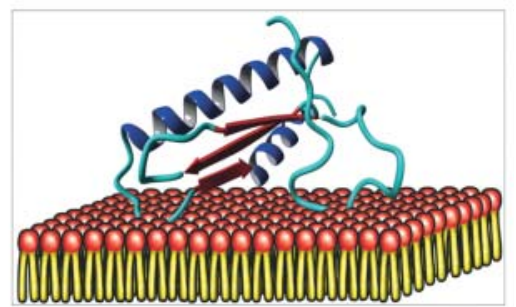

FIGURE 3 a) Immunohistochemical detection of SP-H (surfactant protein H) (red, AEC (3-amino-9-ethylcarbazole) staining) in lung tissue. Bronchioles: cytoplasmic protein distribution in bronchioles (panels 1-4); alveolar duct: protein distribution as a superficial layer (panels 5-6); alveoli: detection in the membrane of alveolar type II cells (panels 7-10). Insets show magnifications. Panels $1,3,5,7$ and 9 scale bars $=100 \mu$ m; panels $2,4,6,8$ and 10 scale bars $=50 \mu \mathrm{m}$. b) Immunofluorescence of lung cell line A549 showing localisation of SP-H within the membrane (SP-H: green; DAPI (4',6-diamidino-2-phenylindole): blue). Scale bar $=20 \mu \mathrm{m}$. c) Immunogold electron microscopy of bronchial epithelium. Positive antibody reactivity is indicated by black dots and red arrows, especially on cilia. Scale bar $=1 \mu \mathrm{m}$. d) Simplified representation of the SP-H protein model interacting with the surface of a lipid monolayer after a 50 ns molecular dynamics simulation. 
antibodies (red arrows and black dots in figure 3c). This indicates that SP-H remains cytoplasmic because of its physicochemical properties and is secreted after modification. In theoretical studies, SP-H showed palmitoylation potential and, if this is the case, it would be able to interact with a lipid membrane similarly to SP-B and SP-C [20]. To determine if SP-H can stabilise the liquid surface, further functional studies are needed. However, these experiments support the results of the MD simulations, which showed theoretical interaction potential for SP-H with a lipid system (fig. 3d).

\section{Influence of cytokines and lipopolysaccharide on SP-H production by A549 as well as SP-H expression in case of bacterial sepsis and asthma}

Because it is difficult to obtain and cultivate primary lung cells, A549 cells were used and incubated to investigate whether SP-H is regulated during infections. This cell line is well established and known to produce surfactant proteins in response to stimulation with lipopolysaccharide (LPS) of the bacterial membrane wall. A significant increase in SP-H mRNA concentration could be detected after 3-24 h stimulation with LPS (fig. 4a). By contrast, the dose-response analysis with interleukin (IL)-1 $\beta$ showed a downregulation of SP-H expression (fig. 4b). Similarly, IL-23 downregulated SP-H mRNA in A549 cells (fig. 4c). The immunohistochemical fluorescence analysis revealed similar results (fig. $4 \mathrm{~d}$ ). The stimulation with IL-1 $\beta$, IL-23 and both IL- $1 \beta$ and IL-23 showed a weak signal for SP-H protein within the cytoplasm and on the endoplasmic reticulum. In contrast, SP-H could not be detected on the cell membrane but within the nucleolus (green spots in fig. 4d). Within tissue from patients suffering from asthma or bacterial sepsis SP-H could be detected especially in alveolar cells (I and II) as well as in alveolar macrophages and the cytoplasm of the epithelial cells.

The subcellular distribution is comparable to the known SPs, namely as granular structures within the cytosol of the epithelial cells $[9,21]$. Moreover, the lung tissue of patients suffering from bacterial sepsis showed an increased expression of SP-H corresponding to the real-time RT-PCR experiment shown in figure 4e. By contrast, the expression of SP-H is decreased in tissue affected by asthma, also corresponding to the real-time RT-PCR experiments shown in figure 4e.

Abnormal accumulation of surfactant has been described in humans since 1958 as a pulmonary disorder called pulmonary alveolar proteinosis, which is associated with accumulation of surfactant lipids and proteins in the airspaces [22]. Abnormal surfactant catabolism by alveolar macrophages could contribute to this disorder [21]. Considering that patients with pulmonary alveolar proteinosis have an increased risk for viral, bacterial and fungal infections, regulation of SP production by alveolar macrophages and epithelial type II cells is very important to avoid infections.

The present study shows that cytokines IL-1 $\beta$, IL-23 and a combination of both downregulate the expression of SP-H. The addition of LPS, a component of the outer membrane component of Gramnegative bacteria, by contrast, induces an increase in SP-H mRNA concentration. LPS induces acute pulmonary inflammation and changes in the composition of the surfactant, and type and activation of the lung resident cell population. In response to immune stimulation, alveolar epithelial type II cells can synthesise SPs which maintain the mucosal integrity. In particular, SP-A, the most abundant pulmonary SP, can bind to LPS, viruses and fungal cell wall components. However, overproduction of SPs in the lung might lead to chronic diseases [23]. This could indicate that LPS simultaneously induces inflammatory cytokines like IL-1 $\beta$ and IL-23 and SP-H production by resident alveolar macrophages via Toll-like receptor (TLR) [24]. Thus, Gram-negative bacterial infection induces SP-H production by alveolar epithelial cells and inflammatory cytokines like IL-1 $\beta$ and IL-23 produced by alveolar macrophages limit this process (fig. 5). The downregulation of SP-H mRNAs caused by proinflammatory cytokines could play an important role during the pathogenesis of respiratory infection. As shown by others [25] and us, cytokineinduced changes in SP mRNA are reflected in the levels of the proteins. In small premature infants developing chronic lung disease [26] and in acute respiratory distress syndrome (ARDS) [27], proinflammatory cytokines are increased in the airways. The surfactant defects evident in chronic lung disease and in ARDS include deficiencies in SP-B [28] and SP-A [29]. It is known that the regulation of SPs by microbial proteins is mediated by cytokines [25].

TLRs are type I transmembrane proteins. In alveolar epithelial cells, LPS can specifically activate TLR4, which then activates mitogen-activated protein kinase family proteins, nuclear factor (NF)- $\mathrm{\kappa B}$ and activator protein (AP)-1 [30]. Both IL-23 and IL-1 $\beta$ activate NF- $\kappa B$ via the canonical pathway involving degradation of $\mathrm{I} \kappa \mathrm{B}$. Aberrant activation of NF- $\mathrm{KB}$ has been linked to inflammatory and autoimmune diseases, septic shock, viral infection and improper immune function and plays a role in cancer development. It is, thus, possible that inflammation has a protective effect to limit an LPS-induced overproduction of SPs by alveolar epithelial type II cells. Understanding of the basic mechanisms of SP-A, -D, -G and -H action might lead to new therapeutic strategies for the cure of several lung diseases. 

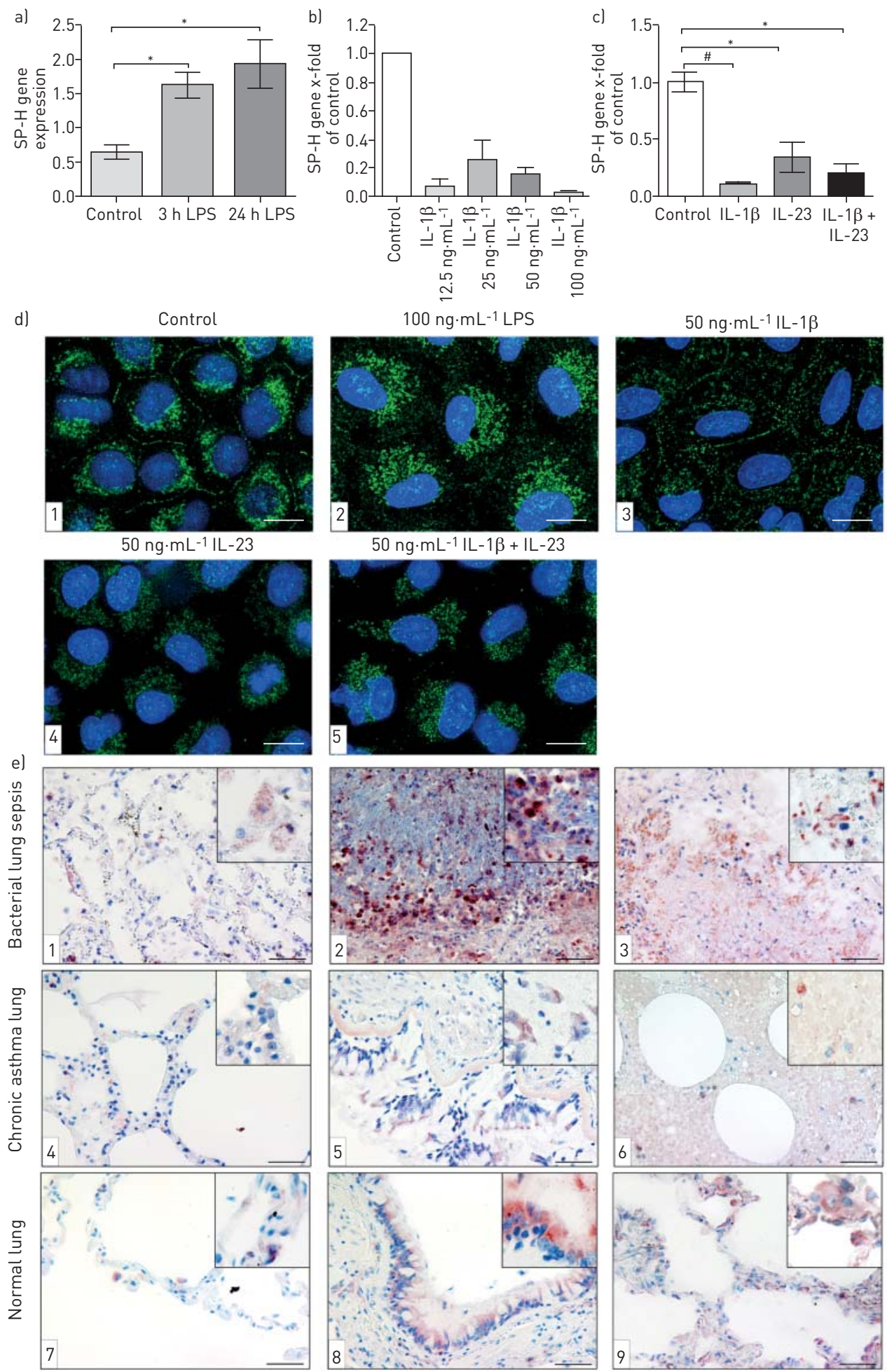

FIGURE 4 Immunoregulation of SP-H (surfactant protein H): real-time RT-PCR on human lung alveolar type II cell line A549. a) Stimulation for 3 and $24 \mathrm{~h}$ with $100 \mathrm{ng} \cdot \mathrm{mL}^{-1}$ lipopolysaccharide (LPS). b) Stimulation for $24 \mathrm{~h}$ with $12.5,25$, 50 and $100 \mathrm{ng} \cdot \mathrm{mL}^{-1}$ of recombinant interleukin (IL)-1 $\beta$. c) Stimulation for $24 \mathrm{~h}$ with $50 \mathrm{ng} \cdot \mathrm{mL}^{-1}$ of IL-1 $\beta$, IL-23 and both. The regulation of SP-H transcript levels was expressed as mean \pm SEM, $n=3$. ${ }^{*}: \mathrm{p} \leqslant 0.05 ;{ }^{*}: \mathrm{p} \leqslant 0.005$. d) Immunofluorescence with anti-SP-H antibody in A549 cells showing localisation of SP-H. Cells were stimulated with LPS, IL-1 $\beta$, IL-23, IL-1 $\beta$ + IL23 for 24 h. SP-H: green; DAPI (4',6-diamidino-2-phenylindole): blue. Scale bars: $10 \mu \mathrm{m}$. e) Detection of SP-H (red, AEC (3-amino-9-ethylcarbazole) staining) in lung diseases: bacterial sepsis (panels 1-3), asthma (panels 4-6) and normal lung (panels 7-9). Regional effects in pathological diseases (panels 2, 3, 5 and 6). Insets show magnifications of the selected regions. Scale bars $=50 \mu \mathrm{m}$. 


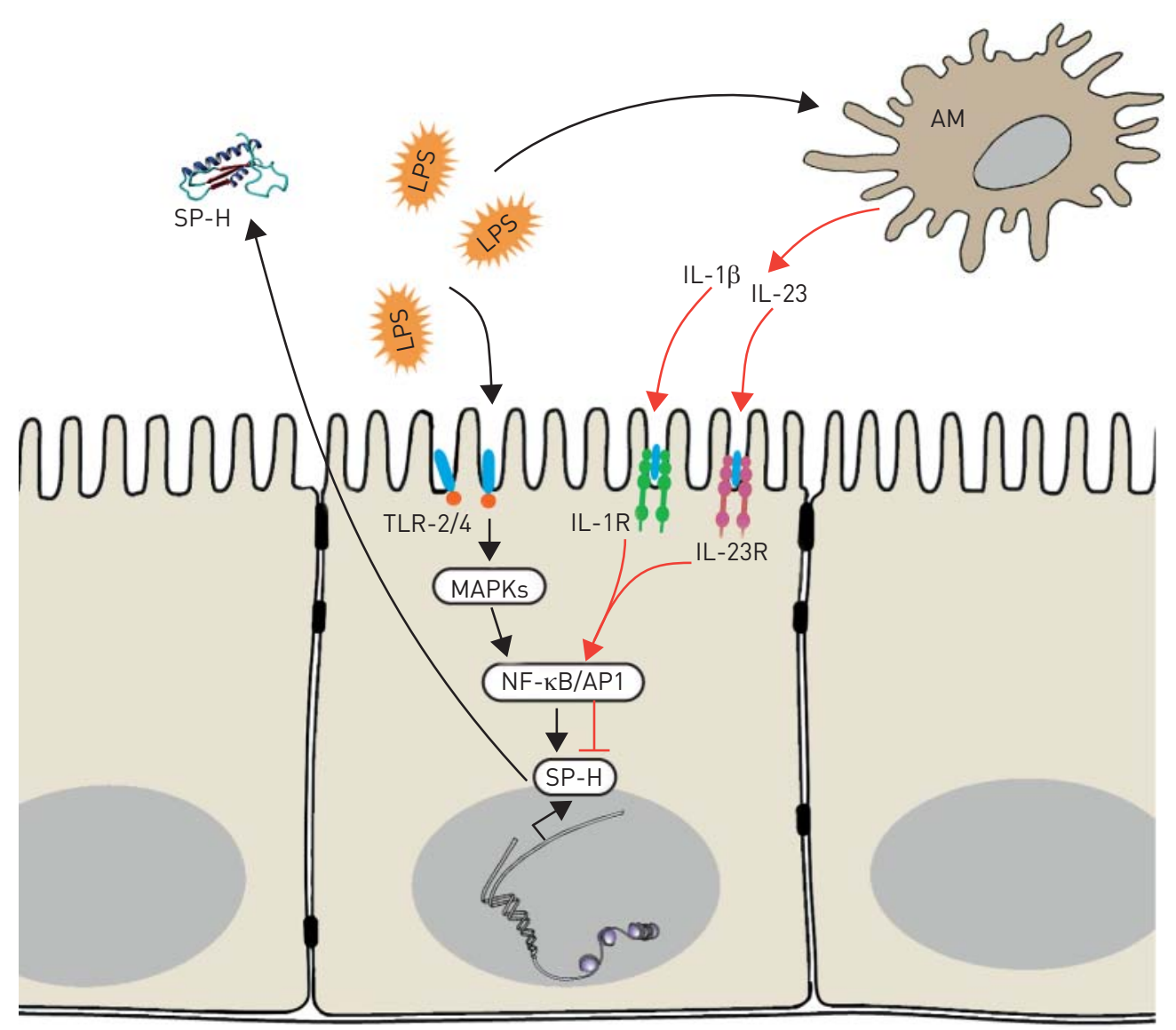

FIGURE 5 Regulation of SP-H (surfactant protein H). The bacterial lipopolysaccharide (LPS) induces the expression of $\mathrm{SP}-\mathrm{H}$ via mitogen-activated protein kinase (MAPK) signalling and nuclear transcription factors (nuclear factor (NF)- $\mathrm{\kappa B}$ and activator protein (AP)-1). In addition, LPS induces production of interleukin (IL)-1 $\beta$ and IL-23 by alveolar macrophages that limit SP-H production by alveolar type II cells. IL-1R: IL-1 receptor; IL-23R: IL-23 receptor; TLR-2/4: Toll-like receptor 2/4; AM: alveolar macrophage.

\section{Conclusion}

We detected and characterised SP-H (SFTA3), a novel secretory surfactant protein expressed and secreted by different human cells and tissues (especially the lung, but also for example kidney and testis), for the first time. The proposed physicochemical properties of SP-H show similarity to the known SP-B and SP-C. In this context the protein modelling studies and molecular dynamics simulations performed indicate surface activity of SP-H. Despite the fact that no structurally conserved immune regulatory domains could be identified in SP-H, this protein might play an important role during inflammation processes and immune defence. SP-H seems to be a new lung surfactant protein with different functions and properties. Based on the current knowledge about the four known surfactant proteins, SP-G and SP-H are the only two novel proteins considered to be SPs. A protein formerly named SP-J proved to be a claudin and, therefore, no longer belongs to the "HUGO surfactant proteins" [31]. Nevertheless, more proteins with functions similar to the SPs are supposed to exist and be part of interfaces and surfaces.

\section{Acknowledgements}

The authors would like to thank S. Beileke, H. Nguyen, A. Fischer, G. Link and J. Pekarsky (Institute of Anatomy, Erlangen, Germany) for excellent technical assistance.

\section{References}

Halliday HL. Surfactants: past, present and future. J Perinatol 2008; 28: Suppl. 1, S47-S56.

Wright JR. Immunoregulatory functions of surfactant proteins. Nat Rev Immunol 2005; 5: 58-68.

Hartshorn KL, Crouch E, White MR, et al. Pulmonary surfactant proteins A and D enhance neutrophil uptake of bacteria. Am J Physiol 1998; 274: L958-L969.

4 Kishore U, Greenhough TJ, Waters P, et al. Surfactant proteins SP-A and SP-D: structure, function and receptors. Mol Immunol 2006; 43: 1293-1315. 
Ding J, Takamoto DY, von Nahmen A, et al. Effects of lung surfactant proteins, SP-B and SP-C, and palmitic acid on monolayer stability. Biophys J 2001; 80: 2262-2272.

6 Cochrane CG, Revak SD. Pulmonary surfactant protein B (SP-B): structure-function relationships. Science 1991; 254: 566-568.

7 Bräuer L, Johl M, Börgermann J, et al. Detection and localization of the hydrophobic surfactant proteins B and C in human tear fluid and the human lacrimal system. Curr Eye Res 2007; 32: 931-938.

8 Bräuer L, Möschter S, Beileke S, et al. Human parotid and submandibular glands express and secrete surfactant proteins A, B, C and D. Histochem Cell Biol 2009; 132: 331-338.

9 Bräuer L, Schicht M, Stengl C, et al. Detection of surfactant proteins A, B, C and D in human gingiva and saliva. Biomed Tech (Berl) 2012; 57: 59-64.

10 Schicht M, Knipping S, Hirt R, et al. Detection of surfactant proteins A, B, C, and D in human nasal mucosa and their regulation in chronic rhinosinusitis with polyps. Am J Rhinol Allergy 2013; 27: 24-29.

11 Yang L, Johansson J, Ridsdale R, et al. Surfactant protein B propeptide contains a saposin-like protein domain with antimicrobial activity at low pH. J Immunol 2010; 184: 975-983.

12 Rausch F, Schicht M, Paulsen F, et al. "SP-G", a putative new surfactant protein - tissue localization and 3D structure. PLoS One 2012; 7: e47789.

13 Wright JR. Clearance and recycling of pulmonary surfactant. Am J Physiol 1990; 259: L1-L12.

14 Heilig R, Eckenberg R, Petit JL, et al. The DNA sequence and analysis of human chromosome 14. Nature 2003; 421: 601-607.

15 Reppert S, Boross I, Koslowski M, et al. A role for T-bet-mediated tumour immune surveillance in anti-IL-17A treatment of lung cancer. Nat Commun 2011; 2: 600.

16 Wright JM, Merlo CA, Reynolds JB, et al. Respiratory epithelial gene expression in patients with mild and severe cystic fibrosis lung disease. Am J Respir Cell Mol Biol 2006; 35: 327-336.

17 Noah TL, Murphy PC, Alink JJ, et al. Bronchoalveolar lavage fluid surfactant protein-A and surfactant protein-D are inversely related to inflammation in early cystic fibrosis. Am J Respir Crit Care Med 2003; 168: 685-691.

18 Barnett CP, Mencel JJ, Gecz J, et al. Choreoathetosis, congenital hypothyroidism and neonatal respiratory distress syndrome with intact NKX2-1. Am J Med Genet A 2012; 158A: 3168-3173.

19 Madsen J, Kliem A, Tornøe I, et al. Localization of lung surfactant protein D on mucosal surfaces in human tissues. J Immunol 2000; 164: 5866-5870.

20 Parra E, Alcaraz A, Cruz A, et al. Hydrophobic pulmonary surfactant proteins SP-B and SP-C induce pore formation in planar lipid membranes: evidence for proteolipid pores. Biophys J 2013; 104: 146-155.

21 Golde DW, Territo M, Finley TN, et al. Defective lung macrophages in pulmonary alveolar proteinosis. Ann Intern Med 1976; 85: 304-309.

22 Rosen SH, Castleman B, Liebow AA. Pulmonary alveolar proteinosis. N Engl J Med 1958; 258 : $1123-1142$.

23 McCormack FX, Whitsett JA. The pulmonary collectins, SP-A and SP-D, orchestrate innate immunity in the lung. J Clin Invest 2002; 109: 707-712.

24 Muzio M, Bosisio D, Polentarutti N, et al. Differential expression and regulation of toll-like receptors (TLR) in human leukocytes: selective expression of TLR3 in dendritic cells. J Immunol 2000; 164: 5998-6004.

25 Väyrynen O, Glumoff V, Hallman M. Regulation of surfactant proteins by LPS and proinflammatory cytokines in fetal and newborn lung. Am J Physiol Lung Cell Mol Physiol 2002; 282: L803-L810.

26 Speer CP. Inflammatory mechanisms in neonatal chronic lung disease. Eur J Pediatr 1999; 158: Suppl. 1, S18-S22.

27 Hybertson BM, Lee YM, Repine JE. Phagocytes and acute lung injury: dual roles for interleukin-1. Ann N Y Acad Sci 1997; 832: 266-273.

28 Gregory TJ, Longmore WJ, Moxley MA, et al. Surfactant chemical composition and biophysical activity in acute respiratory distress syndrome. J Clin Invest 1991; 88: 1976-1981.

29 Hallman M, Lappalainen U, Bry K. Clearance of intra-amniotic lung surfactant: uptake and utilization by the fetal rabbit lung. Am J Physiol 1997; 273: L55-L63.

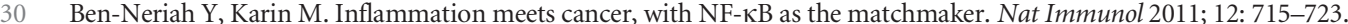

31 Niimi T, Nagashima K, Ward JM, et al. Claudin-18, a novel downstream target gene for the T/EBP/NKX2.1 homeodomain transcription factor, encodes lung- and stomach-specific isoforms through alternative splicing. Mol Cell Biol 2001; 21: 7380-7390. 